\title{
Conhecimento sobre teorias e processo de enfermagem utilizando técnica de associação livre de palavras
}

\author{
Knowledge about theories and nursing process using free word association technique
}

Conocimiento sobre teorías y procesos de enfermería mediante la técnica de asociación de palabras libre

Edficher Margotti ${ }^{1 *}$, Andressa Tavares Parente ${ }^{1}$, Thaianny Cristina Sarmento Gomes ${ }^{1}$, Adriele Cristine Sacramento da Silva ${ }^{1}$, Clara Alice Monteiro Soranso ${ }^{1}$, Anne Letice Soares Braga ${ }^{1}$, Maria Luiza Maués de Sena1, Arthur Filocreão dos Santos Oliveira1', Lorena Gomes de Araújo', Janaina Mendes de Melo'1.

\section{RESUMO}

Objetivo: Descrever o conhecimento de estudantes de enfermagem sobre as teorias de enfermagem e o Processo de Enfermagem. Métodos: Estudo descritivo com abordagem qualitativa, em uma instituição de ensino superior pública, com estudantes do sexto semestre da faculdade de enfermagem, durante uma oficina sobre Processo de Enfermagem, em um cenário de prática que é referência estadual em saúde da mulher e da criança, em novembro de 2019. O formulário online utilizado para coleta dos dados utilizou à Técnica de Associação Livre de Palavras. Os termos evocados foram teorias de enfermagem e processo de enfermagem. Os termos citados foram organizados em planilhas e analisados pelo software IRAMUTEQ®. Resultados: Os termos mais citados para o Processo de Enfermagem foram: coleta de dados, diagnóstico de enfermagem, implementação, histórico de enfermagem, planejamento, intervenção e avaliação. E os mais citados para as Teorias de Enfermagem foram: Autocuidado, Necessidades Humanas Básicas, Transcultural e Ambientalista. Conclusão: Os estudantes possuem conhecimento limitado em relação às diversas teorias de enfermagem, e muitos desconhecem as etapas do Processo de Enfermagem.

Palavras-chave: Cuidados de enfermagem, Diagnósticos de enfermagem, Processos de enfermagem, Enfermagem, Estudantes de enfermagem.

\section{ABSTRACT}

Objective: To describe the knowledge of nursing students about nursing theories and the Nursing Process. Methods: Descriptive study with a qualitative approach, in a public higher education institution, with students from the sixth semester of the faculty of nursing, during a workshop on the Nursing Process, in a practice setting that is a state reference in women's and children's health, in November 2019. The online form used for data collection used the Free Word Association Technique. The terms evoked were nursing theories and nursing process. The terms cited were organized in spreadsheets and analyzed using the IRAMUTEQ® software. Results: The most cited terms for the Nursing Process were: data collection, nursing diagnosis, implementation, nursing history, planning, intervention and evaluation. And the most cited for Nursing Theories were: Self-care, Basic Human Needs, Transcultural and Environmentalist. Conclusion: Students have limited knowledge in relation to various nursing theories, and many are unaware of the stages of the Nursing Process.

Keywords: Nursing care, Nursing diagnosis, Nursing process, Nursing, Students nursing.

1 Universidade Federal do Pará (UFPA), Belém - PA. “E-mail: edficher@ufpa.br 


\section{RESUMEN}

Objetivo: Describir los conocimientos de los estudiantes de enfermería sobre las teorías de enfermería y el proceso de enfermería. Métodos: Estudio descriptivo con abordaje cualitativo, en una institución pública de educación superior, con estudiantes del sexto semestre de la facultad de enfermería, durante un taller sobre el Proceso de Enfermería, en un local de práctica que es un referente estatal en la salud de la mujer y el niño, en noviembre de 2019. El formulario en línea utilizado para la recolección de datos utilizó la Técnica de Asociación de Palabras Libres. Los términos evocados fueron teorías de enfermería y proceso de enfermería. Los términos citados se organizaron en hojas de cálculo y se analizaron con el software IRAMUTEQ®. Resultados: Los términos más citados para el Proceso de Enfermería fueron: recolección de datos, diagnóstico de enfermería, implementación, historia de enfermería, planificación, intervención y evaluación. Y las más citadas por Teorías de Enfermería fueron: Autocuidado, Necesidades Humanas Básicas, Transcultural y Ambientalista. Conclusión: Los estudiantes tienen conocimientos limitados en relación a diversas teorías de enfermería y muchos desconocen las etapas del Proceso de Enfermería.

Palabras clave: Atención de enfermería, Diagnóstico de enfermería, Procesos de enfermería, Enfermería, Estudiantes de enfermería.

\section{INTRODUÇÃO}

A enfermagem surgiu como profissão a partir da necessidade de se estabelecer cuidado às pessoas doentes, construindo assim um perfil de cuidado que caracteriza a profissão, essa prática emerge inerente a necessidade de organizar as atividades e ações prestadas pelo profissional, além da influência das mudanças globais sobre os processos de trabalho de enfermagem que exigem o surgimento de tecnologias que sejam o arcabouço para tais práticas (SIEWERT JS, et al., 2017).

Para sustentar essas necessidades podemos analisar a criação das teorias de enfermagem ao longo dos anos, iniciando por Florence Nightingale a qual propõe com sua teoria Ambientalista, apresentada em 1859, que o ambiente influencia na recuperação e manutenção da saúde do indivíduo doente, pois ele faz parte do meio, logo o equilíbrio ou falta dele estão diretamente ligados à recuperação ou piora do paciente, sendo uma das atividades do profissional enfermeiro monitorar o meio, no qual este indivíduo está inserido (BORSON LAMG, et al., 2018).

A teoria das Necessidades Humanas Básicas (NHB), de 1960, idealizada por Wanda de Aguiar Horta, evidencia a Sistematização da Assistência de Enfermagem (SAE) na aplicação do cuidado, pois considera a enfermagem como a arte do cuidar. Essa teoria está alicerçada em um tripé formado por três leis: a lei do equilíbrio, o universo em seu todo se mantém através do equilíbrio entre os seres, a lei da adaptação, onde estes seres se adaptam a partir do contato com o meio externo e a lei do holismo que considera o ser como um todo inerente a sua existência (CORREIA SR, et al., 2017).

Outra teoria que sustenta a importância da aplicação de instrumentos na prática do enfermeiro é a teoria Transcultural consolidada em 1980 por Madeleine Leininger, pois considera o ser e sua pluralidade, além dos aspectos sociais, culturais e econômicos. Evidencia também que o processo do cuidar, considerado a essência da enfermagem, perpassa todas essas condições e encontra-se em constante mudança, exigindo a existência de instrumentos que sustentem a prática do cuidado individual (REIS AT, et al., 2012).

Dorothea Orem em 1991, ao conceber a teoria de Autocuidado, propõe que a importância da relação entre profissional e paciente, apresenta a influência que o ambiente exerce na manutenção e resgate do autocuidado do indivíduo (SANTOS B, et al., 2017).

Estudos mostram os desafios enfrentados para a utilização do PE no ensino e na prática profissional assistencial e estratégias para implementação do PE no ensino durante a graduação. Ademais, a rotina diária de trabalho nas instituições é difícil, devido à grande demanda de atividades, sobrecarga de trabalho, jornadas exaustivas, desvio de funções e valores dos próprios enfermeiros, que são resistentes à mudança e desvalorização na utilização do método. Se faz importante uma melhor fiscalização por parte dos órgãos responsáveis pelos enfermeiros e um maior comprometimento de gerentes e instituições (TRINDADE LR, et al., 2016). 
Nesse contexto, o Processo de Enfermagem (PE) emerge como um instrumento que tem como objetivo orientar o processo de trabalho do profissional enfermeiro, com o intuito de organizar e sistematizar as ações desse profissional, contribuindo para aplicação de um cuidado individualizado e sistematizado, que permite atender as particularidades e necessidades de cada cliente. As cinco etapas do PE estão pautadas em construir o histórico do paciente, elencar os diagnósticos de enfermagem para o indivíduo assistido, planejar as ações de enfermagem, implementar essas ações e fazer a avaliação para comprovar a eficiência das intervenções propostas, possibilitando ajustes quando forem necessários. Desse modo o PE deve ser sistemático e deliberado tão somente pelo profissional enfermeiro (TRINDADE LR, et al., 2016).

A aplicação adequada do PE respalda cientificamente o cuidado de enfermagem e aproxima o profissional ao indivíduo que necessita de cuidados, dessa forma resgatando os preceitos teóricos de Florence Nightingale acerca da importância do cuidado (SOUZA MFG, 2013).

Para que a atuação da enfermagem se desenvolva e se aprimore, os enfermeiros devem entender que o gerenciamento do cuidado de enfermagem faz parte da gestão do cuidado integral da enfermagem, e devem ser planejadas e executadas em todas as fases do cuidado (SIEWERT JS, et al.,2017).

Assim, o objetivo deste estudo foi descrever o conhecimento de estudantes de enfermagem sobre as teorias de enfermagem e o processo de enfermagem.

\section{MÉTODOS}

Estudo descritivo, com abordagem qualitativa, que buscou descrever o conhecimento dos estudantes de enfermagem, sobre as teorias e o processo de enfermagem, utilizando a TALP. O cenário foi uma instituição hospitalar de ensino, localizada no estado do Pará, durante uma oficina sobre PE, em um cenário de prática que é referência estadual em saúde da mulher e da criança. A população do estudo foram os estudantes do sexto semestre da faculdade de Enfermagem, de uma universidade pública do estado do Pará.

O problema da pesquisa: qual é o conhecimento dos estudantes de enfermagem do sexto semestre, acerca das teorias de enfermagem e o processo de enfermagem utilizando a Técnica de Associação Livre de Palavras (TALP)?

Os critérios de inclusão dos participantes na pesquisa foram: estudantes do sexto semestre curricular, estudantes que iniciariam a prática no cenário hospitalar, referente a atividade curricular de enfermagem pediátrica. Os critérios de exclusão: estudantes do primeiro ao quinto e do sétimo ao nono semestre curriculares, estudantes que não estivessem cursando a atividade curricular de enfermagem pediátrica.

A coleta de dados foi realizada com a ajuda de duas alunas da faculdade de enfermagem, monitoras da atividade curricular de enfermagem pediátrica, e ocorreu através da distribuição de um formulário online, utilizando a TALP, no período da manhã, no dia 02 de novembro de 2019.

A TALP é uma estratégia utilizada para captar a subjetividade de um determinado grupo, em relação a um tema, desta forma, a TALP expõe o núcleo central e periférico de conteúdos implícitos no discurso. Essa exposição é adquirida após o disparo de um estímulo (NOVAIS S, et al., 2020). As variáveis coletadas no formulário, que aqui iremos chamar de estímulos utilizados, foram: teorias de enfermagem (citar três teorias) e processo de enfermagem (citar cinco termos ou palavras que remetesse ao processo de enfermagem), todas numeradas em sequência.

Inicialmente foram solicitados cinco termos para cada evocação, porém foi solicitado, por parte dos estudantes, que houvesse uma diminuição dos termos citados nas teorias de enfermagem, sendo a solicitação atendida e foi diminuído para três termos solicitados.

Primeiramente foi realizado o acolhimento e a apresentação do cenário de prática hospitalar aos estudantes de enfermagem; em seguida, foi realizada uma oficina sobre a sistematização da assistência de enfermagem para os mesmos, e no terceiro momento, foi distribuído o formulário online para a coleta dos termos evocados. 
Os termos evocados foram organizados em planilha no Microsoft Office Professional Plus 2016, e submetido à análise do software IRAMUTEQ® (Interface de $R$ pour les Analyses Mutidimensionnelles de Testes et de Questionnaires) versão 0.7 alpha 2. Este software gratuito realiza múltiplas análises estatísticas sobre textos, denominados corpus textuais, e sobre tabelas com indivíduos ou palavras.

O método selecionado para a interpretação dos dados foi a análise de matriz, este método permite que sejam analisadas listas de palavras, neste caso, as análises realizadas pelo software são, contagem de frequência, análise prototípica e também análise de similitude (CAMARGO BV e JUSTO AM, 2013). A matriz elaborada foi analisada por meio da análise de similitudes relacionadas às teorias de enfermagem, este valor representa 3 termos descritos por cada um dos participantes, demonstrando 36 evocações. Quanto aos termos relacionados ao Processo de Enfermagem, obteve-se o total de 60 termos, este valor representa 5 termos descritos por cada participante.

Os aspectos éticos foram respeitados, seguindo todos os princípios éticos estabelecidos pela Resolução 466 de 2012 e a Resolução 510 de 2016, do Conselho Nacional de Saúde, do Ministério da Saúde. O projeto foi aprovado no Comitê de Ética em Pesquisa da Universidade Federal do Pará (UFPA), sob o número 4.791.348, tendo o Certificado de Apresentação de Apreciação Ética-CAAE no 47348721.2.0000.0018.

\section{RESULTADOS}

Participaram da atividade doze estudantes de enfermagem do sexto semestre da faculdade de enfermagem. A síntese dos resultados obtidos por meio da análise realizada no software IRAMUTEQ $R$ encontra-se descrita no Quadro 1. Neste quadro consta a descrição das evocações e suas respectivas frequências de citação.

Quadro 1 - Análise de frequência de citações das teorias e dos termos evocados.

\begin{tabular}{|l|c|l|c|}
\hline \multicolumn{2}{|c|}{ Teoria de Enfermagens } & \multicolumn{2}{c|}{ Termos evocativos sobre o PE } \\
(3 evocações para cada acadêmocações para cada acadêmico)
\end{tabular}

Fonte: Margotti E, et al., 2021. 
Os termos coletados através do formulário utilizando a TALP, foram submetidos à análise de similitude no IRAMUTEQ, gerando a figura 1, que corresponde às respostas dadas pelos estudantes acerca das Teorias de Enfermagem, a imagem apresenta como resultado dois núcleos principais que se ramificam, os termos que estão inseridos nesses dois núcleos são "autocuidado" citada 11 vezes (30,56\%), que apresenta uma ramificação com o termo "ambientalista", evocado em 4 momentos $(11,11 \%)$, o outro termo evocado que apresenta o núcleo com maior destaque é a "NHB" evocado 10 vezes $(27,78 \%)$, que apresenta ramificações com os termos "transcultural", citado também 4 vezes (11,11\%), "holística" citados em 3 momentos (8,33\%), e "relações interpessoais" que aparece apenas $1 \mathrm{vez}(2,78 \%)$, outros termos que se encontram na margem são: "adaptação" evocado 2 vezes (5,56\%) e "humanística" citado 1 vez (2,78\%)33 (Figura 1).

Figura 1 - Análise de similitude sobre as Teorias de Enfermagem evocadas utilizando a TALP, submetida ao software IRAMUTEQ®.

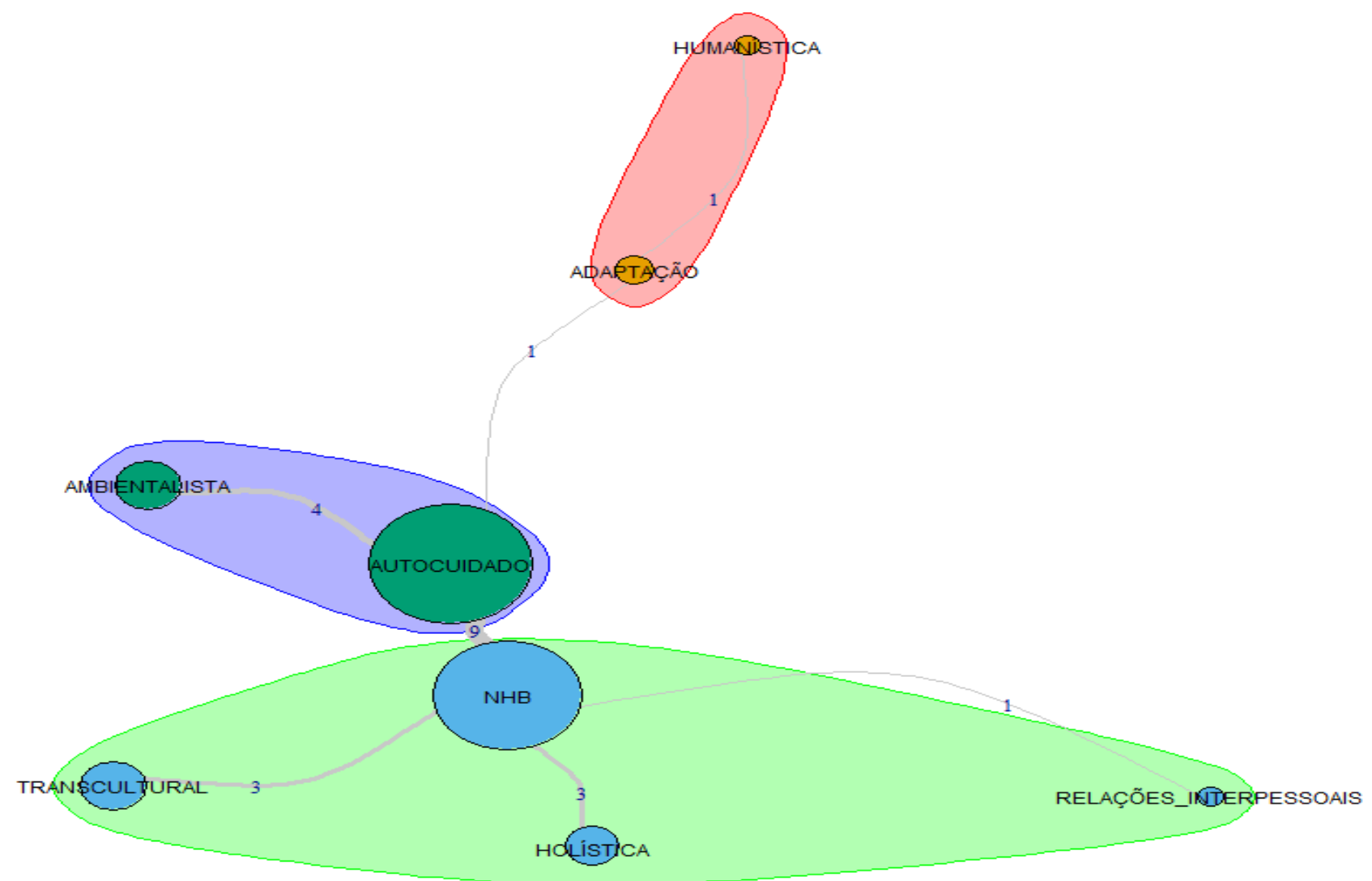

Fonte: Margotti E, et al., 2021.

A próxima análise de similitude gerada pelo software IRAMUTEQ® apresenta quatro grupos de palavras, que se encontram conectadas e possuem frequências distintas, representadas pela dimensão que seus núcleos apresentam, percebe-se que ao evocar o termo "processo de enfermagem", os termos "coleta de dados", "diagnóstico de enfermagem", "planejamento", "implementação" e "histórico de enfermagem", apresentaram núcleos com mais destaque. O termo "coleta de dados" apresenta ramificações que apresentam os termos "avaliação", "valorização", "visão holística" e "plano terapêutico", o termo "diagnóstico de enfermagem" apresenta ligados a ele outros dois termos que apresentam seus núcleos em destaque que são "planejamento" e "implementação", os outros termos ligados a estes três grandes núcleos são "intervenção" e "resultado esperado" (Figura 2).

Para o termo "histórico de enfermagem", temos os termos ramificados "equipe", "sistematização", "assistência" e "modelo assistencial", outros termos que se encontram nas margens são "gerenciamento", "metas" e "problemas" (Figura 2). 
Figura 2 - Análise de similitude sobre os termos evocados para o PE, utilizando a técnica TALP, submetida ao software IRAMUTEQ®.

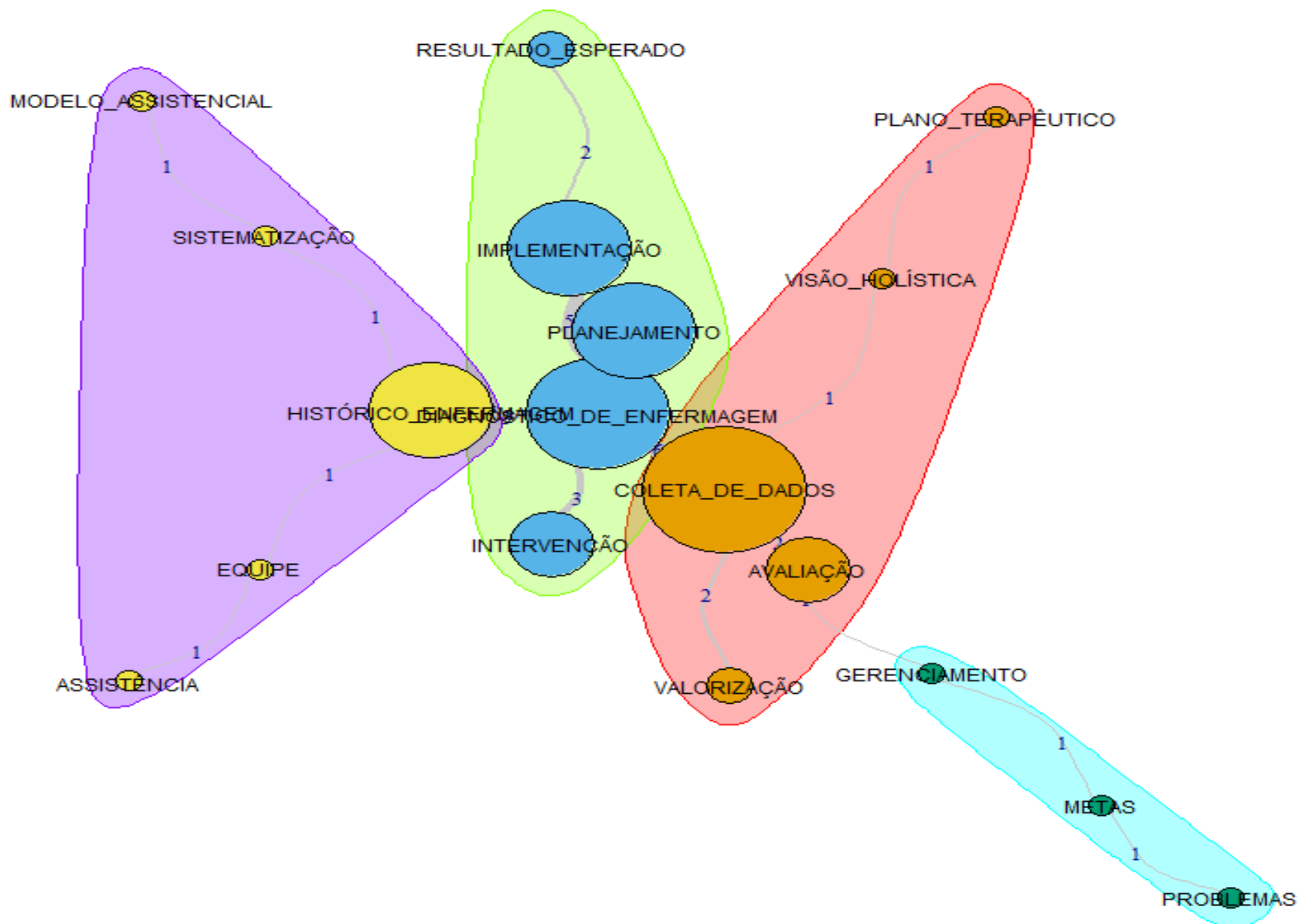

Fonte: Margotti E, et al., 2021.

\section{DISCUSSÃO}

$\mathrm{Na}$ análise de similitude a respeito do conhecimento dos estudantes sobre as teorias de enfermagem, percebeu-se que há uma dificuldade de se falar sobre as teorias, se percebe a pouca variação e limitação do conhecimento sobre as teorias que foram evocadas aos estudantes, cabe ressaltar que a ideia inicial era pedir cinco teorias, porém devido à dificuldade apresentada pela grande maioria, foram pedidas apenas 3 teorias. Esses resultados confirmam os achados da pesquisa realizada em São Paulo que abordou a dificuldade do aprendizado sobre as teorias de enfermagem devido às lacunas existentes na grade curricular das Instituições de Ensino Superior, o que dificulta a aplicação das teorias na prática devido à dificuldade de associar a teoria e prática (SANTOS BP, et al., 2014).

A problemática observada aqui também perpassa aos alunos da pós-graduação, onde os mesmos percebem que o contato com as teorias na graduação tem sido limitado. E reconhecem na pós-graduação uma oportunidade de retomar o conhecimento sobre as teorias de enfermagem, pois isso irá nortear e fortalecer cientificamente a sua prática profissional (MERINO MFGL, 2018).

Nesse contexto é essencial que o processo de formação do enfermeiro favoreça o conhecimento sobre as teorias de enfermagem e seus conceitos, propiciando a aproximação das teorias e a prática. Assim, considerando esses aspectos, seria necessário que esse conteúdo fosse abordado de forma continua perpassando as atividades curriculares, ao invés de ser um processo descontínuo, que dificulta e interfere na assimilação do conhecimento pelos estudantes (SANTOS BP, et al., 2014). 
Durante uma oficina sobre Teorias de Enfermagem desenvolvida em uma universidade federal com alunos

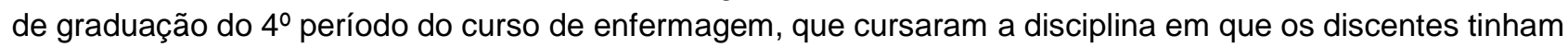
o primeiro contato com essas teorias de enfermagem, levou- se a uma conclusão reflexiva sobre a importância do incentivo e implementação de novas metodologias ativas, possibilitando um repasse de informações mais efetivo a respeito desse tema (ARAÚJO AAC e SILVA GRF 2019).

Com relação às frequências de evocação de cada teoria, foi possível observar que as teorias mais citadas foram as de Autocuidado e a teoria das NHB, as quais são algumas das teorias mais conhecidas, sendo essa última; a teoria mais conhecida e difundida no Brasil (DOURADO SBPB, et al., 2014)

Foi possível observar que os estudantes tentaram citar as cinco etapas do PE em ordem, mas em um dado momento as informações e os conhecimentos dos participantes não foram favoráveis para que eles pudessem elencar todos os passos e na ordem correta. Estes achados corroboram com os resultados de uma pesquisa realizada em Porto Velho, com 23 estudantes de enfermagem de faculdade privada (BARBOSA JMS, 2019), que apresenta as dificuldades que os estudantes de enfermagem possuem em aplicar o PE, pois acabam esquecendo as etapas ou não possuem o conhecimento do PE, apesar da maioria dos estudantes conseguirem conceituá-lo. Nesse mesmo trabalho, verificou-se a dificuldade na aplicação do PE como instrumento de trabalho, isso se deu em parte à formação que não está voltada para a valorização do PE e de suas etapas respectivamente

Desse modo ressalta-se a importância do ensino do PE na graduação, para a formação de enfermeiros mais capacitados, haja vista o valor científico que esta ferramenta traz a prática do profissional, além de direcionar o atendimento ao paciente, para tanto também é necessário que este aluno agregue todos os conhecimentos para a eficiente aplicação do PE, pois também se percebe a falta de êxito em sua aplicação devido ao desconhecimento acerca do exame físico e de fisiopatologia (BARBOSA JMS, et al., 2019).

As questões de diversidade e pluralidade de indivíduos devem ser trabalhadas de maneira mais profunda, durante a formação acadêmica em enfermagem, e fica claro em nossos achados que isso não vem acontecendo, pois parte se do princípio que somos parecidos, mas não somos iguais. Esse entendimento faz perceber o real significado de solidariedade humana. Isso também reflete no cuidado de enfermagem, nem todos requerem os mesmos cuidados (PINTO AC, et al., 2017).

Nossos achados demonstram que os estudantes de enfermagem não compreendem o PE em todas as suas dimensões. Precisam aprender, compreender, e aplicar com precisão. Caso contrário, é inevitável o diagnóstico equivocado, em decorrência, teremos um plano de cuidados ineficaz, intervenções de enfermagem que não serão específicas e avaliações inadequadas e incorretas, ou seja, isso eventualmente levará ao fracasso da assistência de enfermagem. É o que mostra o estudo realizado em uma escola de enfermagem na Turquia, com 155 estudantes, onde foram divididos em dois grupos, um grupo participou efetivamente de um curso sobre terminologias e classificação de enfermagem. O resultado mostrou diferença significativa entre os grupos. Os escores médios dos estudantes que fizeram o curso foram considerados positivos comparados com os estudantes que não fizeram o curso (KARACA T e ASLAN S, 2018).

Além do PE favorecer a autonomia profissional e refletir positivamente no cuidado prestado, mesmo assim percebemos que ainda há algumas fragilidades e dificuldades na aplicação do mesmo. Nosso estudo corrobora com os achados de um trabalho que mostrou o PE como essencial e fundamental para a prática profissional, no que tange a garantia da autonomia e fortalecimento da categoria profissional, porém com fragilidades em aplicar o PE (DORNELES FC, et al., 2021).

Outro estudo com 101 estudantes de enfermagem em Zarga, Jordânia, evidenciou a correta percepção do diagnóstico de enfermagem. No entanto, eles relataram sensação de desconforto durante o uso desses diagnósticos. Embora os estudantes tivessem uma correta percepção do diagnóstico de enfermagem NANDAI, não sabiam distinguir do diagnóstico médico (EL-RAHMAN MA, et al., 2017).

Outro estudo ainda relata que para melhorar a compreensão do uso de diagnósticos de enfermagem, que é uma das etapas do PE, é necessário a introdução de estudos de caso reais, de pacientes e cenários reais. Para os estudantes de enfermagem isso é uma ação considerada, e deve ser apoiada na literatura (KARACA T e ASLAN S, 2018). 
Um outro estudo, com estudantes de enfermagem em Mashhad, Irã, constatou que uma das barreiras mais significativas para a implementação do processo de enfermagem, foi a falta de formação da enfermagem, em específico sobre o processo de enfermagem durante a graduação. $O$ estudo sugeriu que as autoridades do sistema de saúde do país deveriam fazer mudanças nas áreas educacionais, como obtendo aconselhamento e assistência de especialistas na área de enfermagem e tecnologia, e facilitando a implementação do processo de enfermagem no campo de prática (RAJABPOOR M, et al., 2018).

Outro estudo de revisão integrativa, mostrou que o ensino do processo de enfermagem deve ocorrer de forma transversal, envolver estratégias transformadoras e criação de soluções que tragam uma qualificação para o docente de forma contínua, reflexiva, crítica e amarrada com as diretrizes e princípios do Sistema Único de Saúde (SILVA IAS, et al., 2018).

Seguindo essa linha de pensamento, outro trabalho concluiu que o PE constitui um tema que ainda exprime uma grande lacuna entre a formação acadêmica e sua aplicação na prática diária dos acadêmicos. Sendo assim, é de suma importância a conscientização dos docentes para contribuir no conhecimento científico e alinhado ao interesse, à dedicação e à sensibilidade, torna o PE fundamental a uma assistência humanizada (BARBOSA JMS, 2019).

O conhecimento deficiente dos estudantes acerca das teorias de enfermagem existentes e do PE, fez com que diminuíssemos o número de termos que seriam evocados pelos participantes, ou seja, foi diminuído de cinco para três, os termos para a evocação das teorias de enfermagem. Recomenda-se que futuras pesquisas sejam realizadas, incluindo faculdades e universidades de diferentes localizações geográficas e diferentes semestres estudantes. O tamanho da amostra neste estudo foi insuficiente, com base no número total de estudantes matriculados nesta faculdade, um tamanho de amostra maior seria muito mais forte para pesquisas futuras.

\section{CONCLUSÃO}

Observou-se que a maioria dos estudantes de enfermagem possuíam conhecimento acerca das temáticas, visto que os termos mais citados tinham relação com os temas abordados, que foram o PE e as Teorias de enfermagem, porém, a maioria dos estudantes, demonstraram um conhecimento limitado e restrito, não tendo conhecimento das diversas Teorias de Enfermagem e desconhecendo completamente quais são as etapas do $\mathrm{PE}$, não sabendo descrever as cinco etapas do processo, e descrevendo termos e palavras que não tem relação com o PE. Ressalta-se a importância da implantação e implementação de metodologias educativas e interventivas, capazes de identificar conhecimentos comuns e potencializar o ensino aprendizagem desses estudantes com relação ao Processo de Enfermagem.

\section{REFERÊNCIAS}

1. ARAÚJO AAC, SILVA GRF. Office on theory of nursing: successful experience of integration between graduating and post-graduante. Rev Enferm UFPI, 2019; 8(1): 78-82.

2. BARBOSA JMS, et al. O Processo de Enfermagem sob a ótica do acadêmico de uma faculdade privada de Rondônia. Revista Eletrônica Acervo Saúde, 2019; 29: 1-9.

3. BORSON LAMG, et al. A teoria ambientalista de Florence Nightingale. Rev Saúde em Foco, 2018; (10).

4. CAMARGO BV, JUSTO AM. Tutorial para uso do software IRAMUTEQ. Laboratório de psicologia social da comunicação e cognição. 2013.

5. CORREIA SR, et al. Cuidados de Enfermagem prestados à parturiente adolescente sob a luz da Teoria de Wanda Horta Nursing care to adolescent woman in labor in the light of Wanda Horta's theory. R pesq cuid fundam online, 2017; 9(3): 857-66.

6. DORNELES FC, et al. Processo de enfermagem e suas implicações na prática profissional do enfermeiro: revisão integrativa de literatura. Revista Eletrônica Acervo Saúde, 2021; 13(2): e6028.

7. DOURADO SBPB, et al. Conhecimentos e aplicabilidade das teorias de enfermagem pelos estudantes. Rev Enferm UFSM, 2014; 4(2): 284-291.

8. EL-RAHMAN MA, et al. Perceptions and Attitudes Toward NANDA-I Nursing Diagnoses: A Cross-Sectional Study ofJordanian Nursing Students. Journal of Nursing Knowledge, 2017; 28(1): 13-18. 
9. KARACA T, ASLAN S. Effect of 'nursing terminologies and classifications' course on nursing students' perception of nursing diagnosis. Nurse education today, 2018; 67:114-117.

10. MERINO MFGL, et al. Nursing theories in professional training and practice: perception of postgraduate nursing students. Rev Rene, 2018; 19: e3363.

11. NOVAIS S, et al. A representação social da enfermagem e do ser enfermeiro: perspectiva dos estudantes de enfermagem e dos tutores. Millenium, 2020; 2(13): 51-60.

12. PINTO AC, et al. Conceito de ser humano nas teorias de enfermagem: aproximação com o ensino da condição humana. Pro-Posições, 2017; 28: supl.1.

13. RAJABPOOR $\mathrm{M}$, et al. Barreiras à implementação do processo de enfermagem do ponto de vista de docentes, gerentes de enfermagem, enfermeiras e estudantes de enfermagem. J Holist Nurs Midwifery, 2018; 28(2): $137-142$.

14. REIS AT, et al. O cuidado à mulher na contemporaneidade: reflexões teóricas para o exercício da enfermagem transcultural. Rev Min Enferm, 2012; 16(1): 129-135.

15. SANTOS B, et al. Da formação à prática: importância das teorias do autocuidado no processo de enfermagem para a melhoria dos cuidados. Journal of Aging \& Innovation, 2017; 6(1): 51-54.

16. SANTOS BP, et al. Formação e práxis do enfermeiro à luz das teorias de enfermagem. Rev Bras Enferm., 2019; 72(2): 593-7.

17. SIEWERT JS, et al. Gestão do cuidado integral em enfermagem: reflexões sob a perspectiva do pensamento complexo. Rev Min Enferm, 2017; 21: 1-5.

18. SILVA IAS, et al. O ensino do processo de enfermagem. Revista de enfermagem da UFPE on line., 2018; 12(9): 2470 8.

19. SOUZA MFG, et al. O processo de enfermagem na concepção de profissionais de Enfermagem de um hospital de ensino. Rev Bras Enferm., 2013; 66(2): 167-73.

20. TRINDADE LR, et al. Processo de enfermagem: desafios e estratégias para sua implementação sob a ótica de enfermeiros. Saúde (Santa Maria), 2016; 42(1): 75-82. 